\title{
BMJ Open Pharmacological interventions for prevention and management of delirium in intensive care patients: a systematic overview of reviews and meta-analyses
}

\author{
Marija Barbateskovic, ${ }^{1,2}$ Sara Russo Krauss, ${ }^{1}$ Marie Oxenboell Collet, ${ }^{2,3}$ \\ Laura Krone Larsen, ${ }^{4}$ Janus Christian Jakobsen, ${ }^{1,2,5}$ Anders Perner, ${ }^{2,3}$ \\ Jørn Wetterslev ${ }^{1,2}$
}

To cite: Barbateskovic M, Krauss SR, Collet M0, et al. Pharmacological interventions for prevention and management of delirium in intensive care patients: a systematic overview of reviews and meta-analyses. BMJ Open 2019;9:e024562. doi:10.1136/ bmjopen-2018-024562

- Prepublication history and additional material for this paper are available online. To view these files, please visit the journal online (http://dx.doi. org/10.1136/bmjopen-2018024562).

Received 1 June 2018 Revised 7 September 2018 Accepted 7 December 2018

Check for updates

(C) Author(s) (or their employer(s)) 2019. Re-use permitted under CC BY-NC. No commercial re-use. See rights and permissions. Published by BMJ.

For numbered affiliations see end of article.

Correspondence to

Marija Barbateskovic;

marija.barbateskovic@ctu.dk

\section{ABSTRACT}

Objectives We assessed the evidence from reviews and meta-analyses of randomised clinical trials on the effects of pharmacological prevention and management of delirium in intensive care unit (ICU) patients.

Methods We searched for reviews in July 2017 in: Cochrane Library, MEDLINE, Embase, Science Citation Index, BIOSIS Previews, CINAHL and LILACS. We assessed whether reviews were systematic according to Preferred Reporting Items for Systematic Reviews and MetaAnalyses (PRISMA) and assessed the methodological quality using ROBIS.

Outcome measures Primary outcomes: all-cause mortality, serious adverse events, prevention of delirium and management of delirium. Secondary outcomes: quality of life; non-serious adverse events and cognitive function. Results We included 378 reviews: 369 narrative reviews, eight semisystematic reviews which failed on a maximum of two arbitrary PRISMA criteria and one systematic review fulfilling all 27 PRISMA criteria. For the prevention of delirium, we identified the one systematic review and eight semisystematic reviews all assessing the effects of alpha-2-agonists. None found evidence of a reduction of mortality (systematic review RR $0.99,95 \% \mathrm{Cl} 0.79$ to 1.24). The systematic review and three semisystematic reviews found no evidence of an effect for the prevention of delirium (systematic review RR $0.85,0.63$ to 1.14). Conversely, four semisystematic reviews found a beneficial effect. Serious adverse events, quality of life, non-serious adverse events and cognitive function were not assessed. We did not identify any systematic or semisystematic reviews addressing other pharmacological interventions for the prevention of delirium. For the management of manifest delirium, we did not identify any systematic or semisystematic review assessing any pharmacological agents.

Conclusion Based on systematic reviews, the evidence for the use of pharmacological interventions for prevention or management of delirium is poor or sparse. A systematic review with low risk of bias assessing the effects of pharmacological prevention of delirium and management of manifest delirium in ICU patients is urgently needed.

PROSPERO registration number CRD42016046628.
Strengths and limitations of this study

- We used a transparent and systematic method which followed widely accepted methodological standards.

- We conducted a thorough and comprehensive literature search.

- Preferred Reporting Items for Systematic Reviews and Meta-Analyses was chosen as the gold standard for defining a systematic review.

- We did not search for individual trials or performed meta-analyses and Trial Sequential Analysis within each of the groups of pharmacological agents.

\section{INTRODUCTION}

Delirium is a complex acute organic syndrome characterised by a reduced ability to focus, sustain or shift attention, and either a change in cognition or the development of perceptual disturbances. ${ }^{1}$ Delirium is classified in motoric subtypes: (1) hypoactive delirium; (2) hyperactive delirium and (3) a mixed form delirium. Hypoactive and mixed delirium are most common in intensive care unit (ICU) patients, ${ }^{2}{ }^{3}$ and hypoactive delirium has been suggested to have worse outcomes. ${ }^{4}$ In ICU patients, $25 \%$ to $89 \%$ are reported to be affected by delirium, which is associated with increased mortality in these patients. ${ }^{5-9}$ Furthermore, delirium is associated with increased morbidity, including increased duration of mechanical ventilation, and ICU and hospital length of stay. ${ }^{6}{ }^{10-16}$ Patients with delirium may experience functional decline after ICU discharge and long-term cognitive impairment. ${ }^{11} 1215$

Up-to-date critical care guidelines recommend non-pharmacological strategies in both the prevention and management of manifest delirium. ${ }^{17}$ These strategies may include early mobilisation and reorientation 
of the patient, risk factor assessment and normalisation of the sleep-wake cycle. ${ }^{18}$ When delirium is suspected or identified, guidelines suggest that patients should be evaluated to identify potential underlying causes, allowing for deficiencies to be corrected, or exposures to be removed. Only when non-drug methods have failed to control symptoms should pharmacological interventions be used. ${ }^{1920}$ Nonetheless, a recently performed inception cohort study found that haloperidol was used as management option in $46 \%$ of ICU patients diagnosed with delirium, and dexmedetomidine in $21 \%$. $^{16}$

Pharmacological interventions for delirium have focused on alterations in neurotransmitter pathways, in particular dopaminergic and cholinergic pathways. Several pharmacological strategies have been used against delirium in the ICU patients: antipsychotics; sedatives; cholinesterase inhibitors; opioids; and melatonin and melatonin antagonists. Haloperidol is considered the drug of choice when managing manifest delirium in ICU settings ${ }^{21-25}$ and some international guidelines recommend haloperidol in the management of manifest ICU delirium. ${ }^{1926}{ }^{27}$ However, the two latest iterations of the guideline by the American College of Critical Care Medicine and the Society of Critical Care Medicine no longer recommend managing delirium with haloperidol due to lack of evidence. ${ }^{1728}$ In general, pharmacological interventions are not recommended for the prevention of delirium in ICU patients. ${ }^{196-28}$

Systematic reviews and meta-analyses have become one of the most widely used methods to quantify the effects of medical interventions and are frequently being recognised as the best available evidence for decisions about healthcare management and policy. ${ }^{29} 30$ A preliminary search identified several reviews investigating the effects of pharmacological interventions for the prevention and management of delirium. However, uncertainty regarding the benefits and harms of pharmacological interventions appeared to be considerable, and trials have shown either positive, ${ }^{31} 32$ equipoise ${ }^{33} 34$ or negative results. $^{35}$

The objective of this overview of reviews was to systematically and critically assess the quantity and the quality of the available reviews and meta-analyses of randomised clinical trials on the effects of pharmacological prevention and management of delirium in ICU patients.

\section{METHODS}

We conducted this systematic overview of reviews with a registered (PROSPERO CRD42016046628) and published protocol, ${ }^{36}$ in accordance with the Preferred Reporting Items for Systematic Reviews and Meta-Analyses (PRISMA) (Electronic supplementary material (ESM), table 1). We used the systematic review methods principles outlined in the Cochrane Handbook ${ }^{37}$ and the recommendations given by Robinson et $a l^{38}$

\section{Criteria for considering reviews for inclusion}

We included all reviews and meta-analyses of pharmacological interventions for the prevention of delirium or management of manifest delirium (defined as diagnosed delirium) in adult ICU patients. We predefined a systematic review as a review positively fulfilling the PRISMA reporting guidelines. ${ }^{39}$

We defined adult ICU patients as those treated in an ICU (or similar terms defined by the review authors) of any specialty, for example, medical, surgical, trauma, cardiac. We included reviews of ICU patients aged 18 years or older and included both acute surgery patients and elective cardiac surgery patients.

Table 1 Summary of risk of bias assessment of the single systematic review and the eight semisystematic reviews using ROBIS

\begin{tabular}{|c|c|c|c|c|c|c|}
\hline \multirow[b]{2}{*}{ Review } & \multirow{2}{*}{$\begin{array}{l}\text { Violated } \\
\text { PRISMA } \\
\text { criteria }\end{array}$} & \multicolumn{4}{|l|}{ ROBIS Phase 2} & \multirow{2}{*}{$\begin{array}{l}\text { ROBIS Phase } 3 \\
\text { Overall risk of } \\
\text { bias in the review }\end{array}$} \\
\hline & & $\begin{array}{l}\text { Study eligibility } \\
\text { criteria }\end{array}$ & $\begin{array}{l}\text { Identification and } \\
\text { selection of studies }\end{array}$ & $\begin{array}{l}\text { Data collection and } \\
\text { study appraisal }\end{array}$ & $\begin{array}{l}\text { Synthesis } \\
\text { and findings }\end{array}$ & \\
\hline Lin et $a l^{51}$ & \#5; \#27 & (2) & (2) & (2) & (2) & (2) \\
\hline Fraser $^{52}$ & \#5; \#8 & (2) & (:) & (:) & (2) & (2) \\
\hline Zhang et $a l^{48}$ & \#5 & (:) & (:) & ;) & (2) & (2) \\
\hline Pasin et $a l^{50}$ & \#5; \#27 & (2) & : & ;) & (2) & : \\
\hline Chen et $a l^{46}$ & 0 & (:) & ;) & ;) & (:) & (:) \\
\hline Tran et $a l^{54}$ & \#15; \#22 & ;) & ;) & (:) & (2) & (2) \\
\hline
\end{tabular}

\#4, objectives; \#5, protocol and registration; \#8, search; 15, risk of bias across studies (methods); \#22, risk of bias across studies (results); \#27, funding; $\odot$, low risk; $\odot$, high risk. 
We excluded reviews on ICU patients with delirium caused by alcohol withdrawal, terminally ill patients, patients admitted to emergency departments and elective surgery patients, except cardiac surgery.

Results on all primary and secondary outcomes of the included systematic reviews were a priori planned to be reported. ${ }^{36}$ However, we defined the primary and secondary outcomes in this overview of reviews as follows $^{36}$ :

\section{Primary outcomes}

1. All-cause mortality

2. Proportion of participants with a serious adverse event, defined as an event (experience) or reaction in any untoward medical occurrence that at any dose results in death, is life-threatening, requires prolongation of hospitalisation or results in persistent or significant disability/incapacity ${ }^{40}$

3. Proportion of participants with resolution of delirium symptom at end of treatment (management of delirium) and proportion of participants with delirium despite the administration of a pharmacological agent before being diagnosed with delirium (prevention of delirium)

Secondary outcomes

1. Quality of life as defined by review authors (eg, measured with SF36) ${ }^{41}$

2. Proportion of participants with non-serious adverse events defined as adverse events which are not serious

3. Cognitive function as defined by review authors (eg, measured with Repeatable Battery for the Assessment of Neuropsychological Status ${ }^{42}$ (continuous score)

\section{Search methods for identification of reviews}

We searched the Cochrane Library, MEDLINE (OvidSP), Embase (OvidSP), Science Citation Index-Expanded (Web of Science), BIOSIS Previews (Web of Science), Cumulative Index to Nursing \& Allied Health Literature (CINAHL), Latin American Caribbean Health Sciences Literature (LILACS) and Allied and Complementary Medicine Database (AMED) in July 2017, in order to identify reviews eligible for inclusion. Full search strategies and time spans of the searches are provided in electronic supplementary material-ESM.

\section{Data collection and analysis}

Four authors (MB, SRK, MOC, LKL) independently screened the titles and abstracts of all reports identified in the searches using Covidence and comparison was made within pairs. ${ }^{43}$ Reports deemed potentially relevant by any of the review authors were obtained in full text, and the full-text papers were assessed for eligibility by two review authors independently before being assessed for inclusion and compared within pairs. Disagreements were resolved by consensus. Reviews containing a methods section and/ or a literature search were hereafter checked against the PRISMA criteria. ${ }^{39}$ Initially, it was our intention to only include systematic reviews fulfilling all 27 PRISMA criteria, but we decided pragmatically to define a group of reviews which failed on a maximum of two arbitrary PRISMA criteria as semisystematic reviews.

Four authors (MB, SRK, MOC, LKL) independently extracted predefined data of the included reviews using a data extraction form (supplementary material), which was specifically designed and piloted by the review team, and comparisons were made in pairs.

We extracted the following review characteristics:

1. Review identification: authors, year, title

2. From the systematic review(s), we extracted data on the number of trials included, the number of participants included, ICU population (eg, medical or surgical), diagnostic criteria of delirium, type of pharmacological agent(s) included, primary and secondary outcomes, results on primary and secondary outcomes, type of meta-analytic and sequential analysis used and the authors' conclusion

In addition, for all included reviews and meta-analyses, we extracted information on whether haloperidol was recommended for the management of delirium registered as either 'Yes/No/Not stated'. Disagreements concerning the extracted data were discussed and decision reached between the authors.

\section{Assessment of methodological quality of included reviews}

The methodological quality of the reviews failing on a maximum of two arbitrary PRISMA criteria were hereafter assessed with the ROBIS tool. ${ }^{44}$

\section{Data synthesis}

We a priori ${ }^{36}$ planned to perform meta-analysis and trial sequential analysis ${ }^{45}$ of the trials with overall low risk of bias. However, as we solely identified trials with overall high risk of bias, we did not perform the analyses.

We categorised reviews into:

1. Systematic reviews (a review positively fulfilling all 27 PRISMA criteria) ${ }^{39}$

2. Semisystematic reviews being in overall agreement with the PRISMA statement except failing on a maximum of two arbitrary PRISMA criteria

3. Narrative reviews (any review not fulfilling the criteria for a systematic review or the criteria for a semisystematic review)

For the systematic reviews assessed to be of low risk of bias, two authors (MB, MOC) independently assessed the methodological quality of each included trial with the Cochrane risk of bias tool. ${ }^{37}$ Disagreements were discussed, and agreement was reached between the authors. Results are presented narratively by the indication for use (prevention or/and management), followed by the type of pharmacological agent and the type of outcome.

\section{PATIENTS AND PUBLIC INVOLVEMENT}

Patients and the public were not involved in this research. 


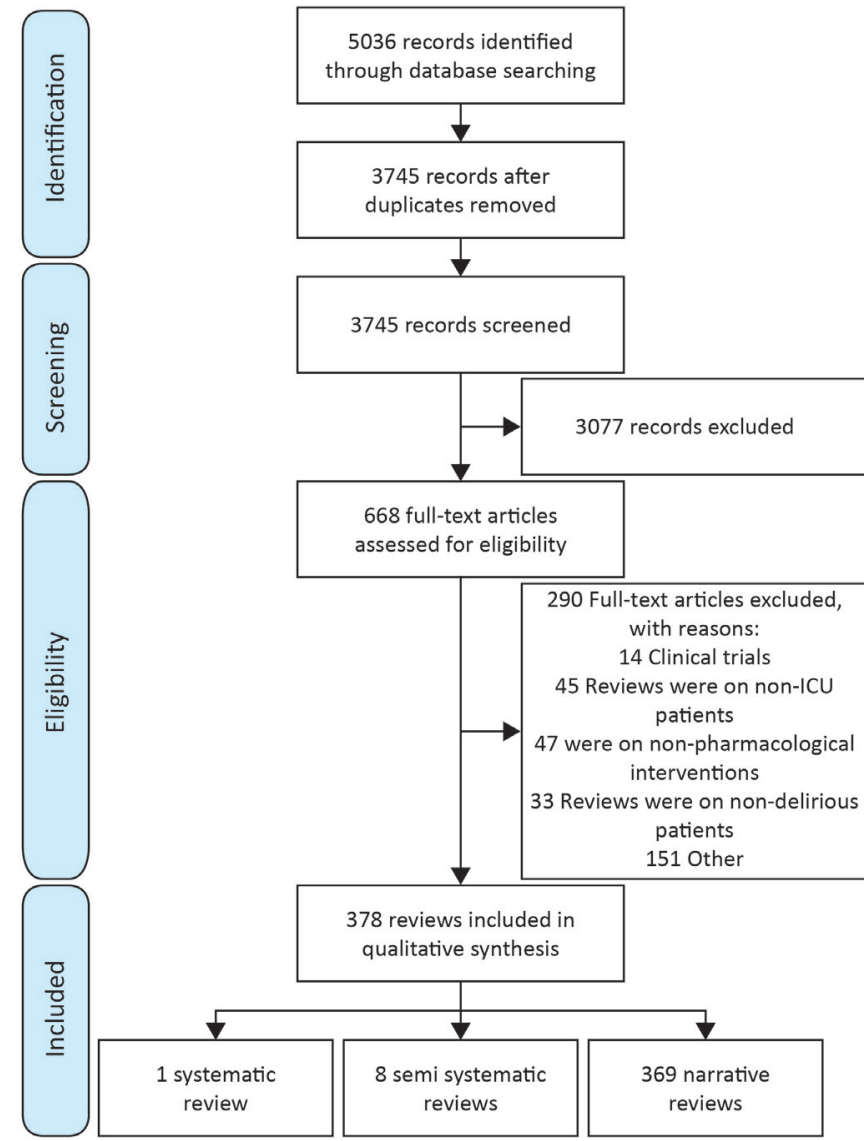

Figure 1 Preferred reporting items for systematic reviews and meta-analyses flowchart.

\section{RESULTS}

We identified 5036 potentially relevant references and finally included 378 reviews (figure 1).

\section{Description of included reviews}

We only identified one systematic review ${ }^{46}$ fulfilling all 27 PRISMA criteria (ESM table 2), eight semisystematic reviews ${ }^{47-54}$ failing on a maximum of two PRISMA criteria and 369 narrative reviews.

\section{The systematic review}

- Chen $e t a t^{46}$ assessed the safety and efficacy of alpha-2 agonists for sedation, compared with traditional sedatives, in mechanically ventilated critically ill patients. This review included seven trials randomising 1624 participants. All included trials investigated adults and compared dexmedetomidine with traditional sedatives (propofol, midazolam or lorazepam).

\section{Semisystematic reviews}

1. Tan $e t a \tilde{l}^{3}$ assessed the effects of using dexmedetomidine as a sedative and analgesic agent compared with placebo or alternative sedative agents, such as propofol and benzodiazepines, in critically ill patients; 24 randomised trials, involving 2419 patients, were included.

2. Lin $e t a{ }^{\tilde{1}}$ assessed the effects of using dexmedetomidine compared with alternative sedative agents fol- lowing cardiac surgery; five randomised trials and six observational studies were included. We report on a subgroup analysis including five randomised trials and a prospective descriptive study.

3. Fraser et $a \tilde{l}^{2}$ reviewed benzodiazepine compared with non-benzodiazepine (four randomised trials with dexmedetomidine and two with propofol) regimens in mechanically ventilated ICU patients. Six randomised trials, involving 1225 patients, were included.

4. Xia $e t a t^{77}$ assessed the influence of dexmedetomidine and propofol on adult ICU sedation. Ten randomised trials, involving 1202 participants, were included.

5. Zhang et $a t^{48}$ included all postoperative trials reporting on delirium risk. We report on only one comparison, alpha-2-adrenoreceptor agonists compared with other sedatives for the risk of postoperative delirium, where only cardiac surgical trials have been included, as the other outcomes included patient groups we excluded (two randomised trials on dexmedetomidine and one on clonidine, involving 445 patients).

6. Pasin et $a \tilde{p}^{0}$ compared dexmedetomidine with any comparator in the ICU setting (nine randomised trials in ICU, four in cardiac surgery and one in cervical spine surgery, including a total of 3029 patients).

7. Tran et a $\tilde{l}^{4}$ assessed alpha-2 agonists (all trials reported on dexmedetomidine) for non-procedural sedation in critically ill brain-injured patients on mechanical ventilation. Both randomised trials and observational studies were included. Six randomised trials including a total of 318 patients were included. However, due to lack of clinical homogeneity of the randomised trials and studies, pooling was deemed inappropriate. We only report on outcomes which were defined a priori.

8. Liu $e t a l^{49}$ compared the effects of dexmedetomidine and propofol sedation in adult patients after cardiac surgery; eight randomised trials involving 969 patients were included.

\section{Risk of bias in the systematic review and the eight}

\section{semisystematic reviews}

We assessed the systematic review by Chen $e t a l^{46}$ as overall low risk of bias (table 1 ).

However, the seven included trials ${ }^{55-60}$ were all overall high risk of bias (figure 2). The eight semisystematic reviews failing on a maximum of two arbitrary PRISMA criteria, by Tan et $a l,^{53}$ Lin $e t a l,{ }^{51}$ Fraser et al, ${ }^{52}$ Xia et $a l,{ }^{47}$ Zhang et al, ${ }^{48}$ Pasin,${ }^{50} \operatorname{Tran}^{54}$ and Liu et al, ${ }^{49}$ were all overall high risk of bias. All 46 trials included in these eight semisystematic reviews were overall high risk of bias.

\section{Effects of pharmacological interventions for delirium in ICU patients}

Prevention of delirium

Antipsychotics

We did not identify any systematic review or semisystematic review assessing the effects of antipsychotics (eg, haloperidol) for the prevention of delirium. 
Table 2 Pooled effect estimates reported by the systematic review and semisystematic reviews by outcome and type of pharmacological agent

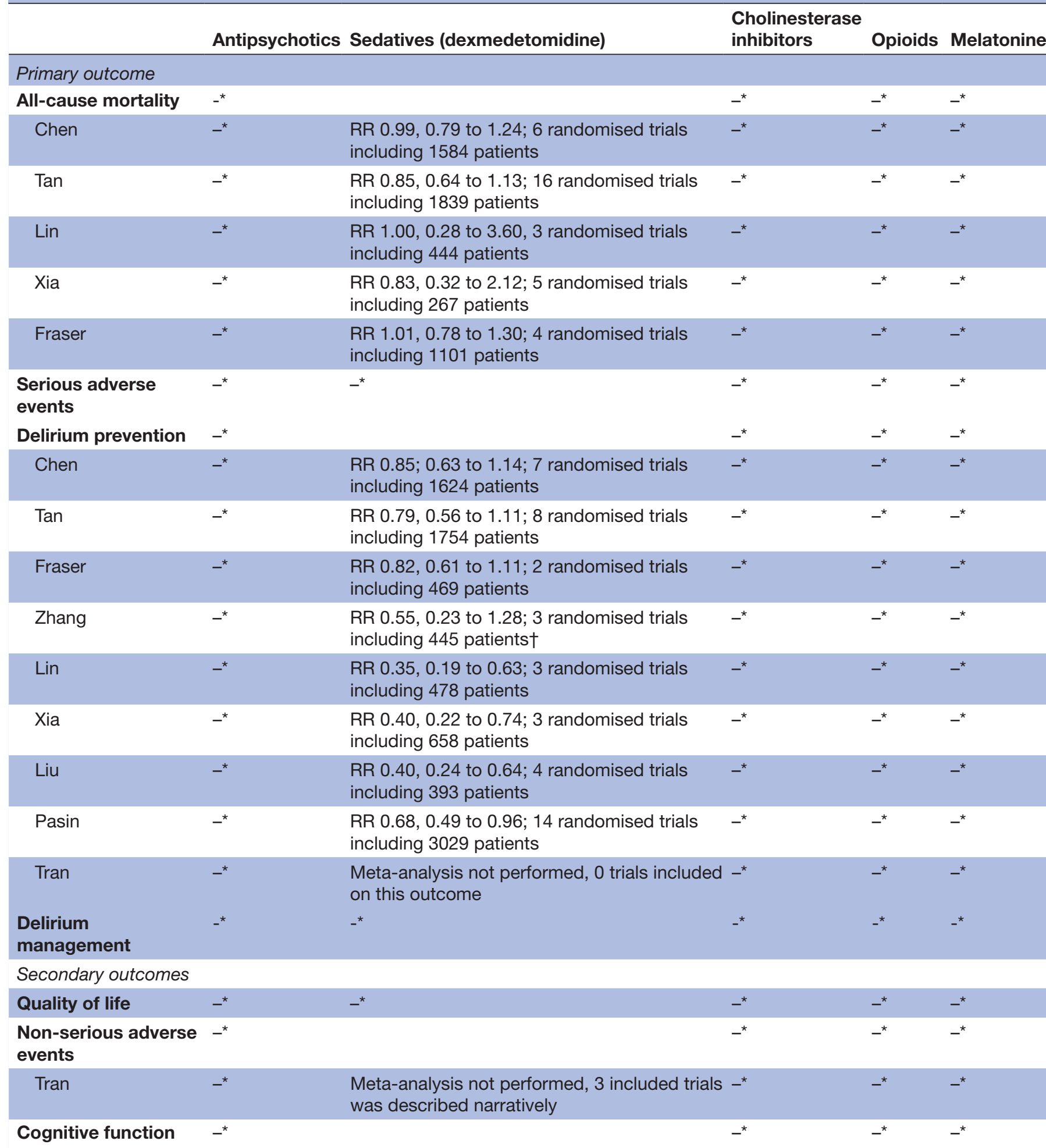

*No systematic review or semisystematic review identified or assessed this outcome.

†Clonidine and dexmedetomidine.

\section{Sedatives}

\section{All-cause mortality}

When assessing mortality (table 2), Chen et $a t^{46}$ did not find evidence for a difference when comparing dexmedetomidine with traditional sedatives (midazolam, lorazepam or propofol).

Neither did Tan et $a \tilde{l}^{\tilde{3}}$ and Lin et $a \tilde{l}^{1}$ when comparing dexmedetomidine with traditional sedatives. Additionally, 


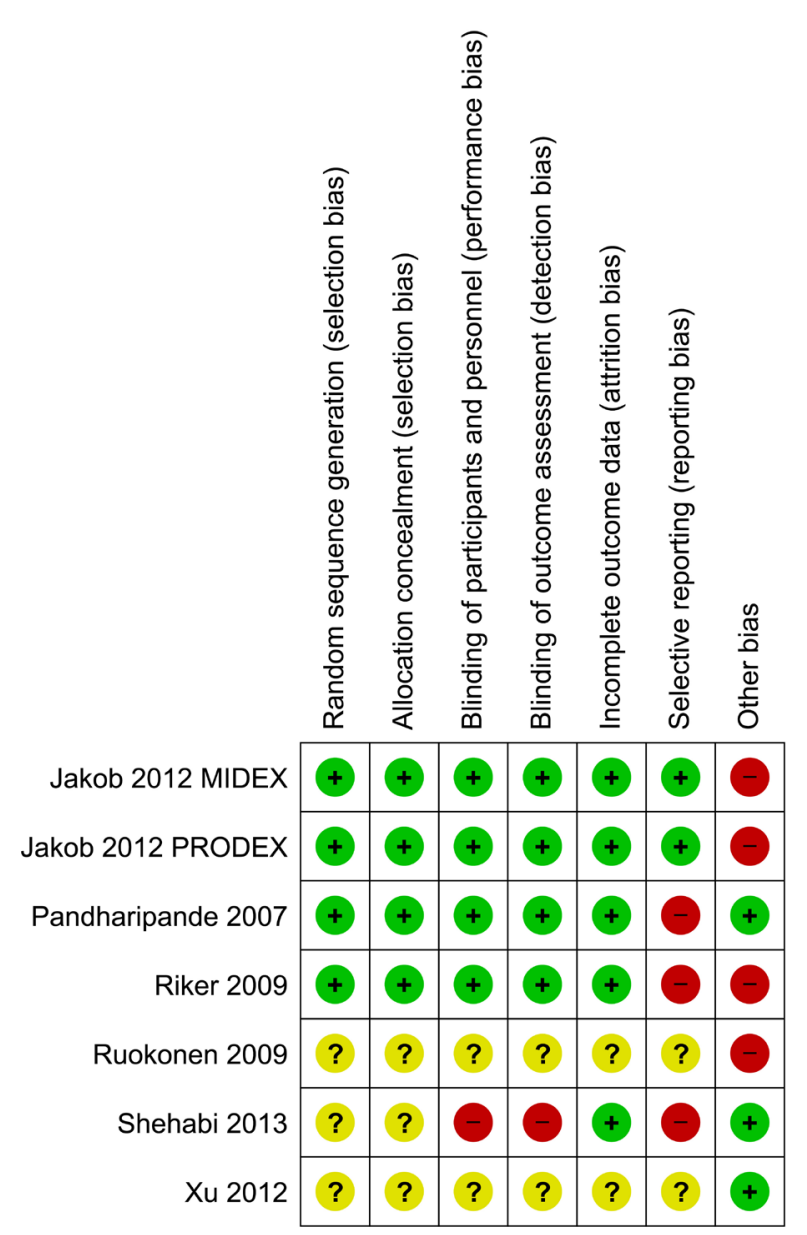

Figure 2 Risk of bias summary: review authors' judgements about each risk of bias item for each included trial in the only included systematic review (Chen 2015).

Xia et $a l^{47}$ compared dexmedetomidine with propofol and also found no difference in mortality. Fraser et $a \tilde{l}^{2}$ compared benzodiazepines with non-benzodiazepines (dexmedetomidine or propofol) and found no difference in mortality.

\section{Serious adverse events}

We did not identify any systematic review or semisystematic review assessing the effects of sedatives on risk of serious adverse events.

\section{Risk of delirium}

When assessing the effect of prophylactic use of alpha-2-agonists compared with alternative sedatives on the subsequent risk of delirium (table 2), the systematic review (on dexmedetomidine $)^{46}$ and three semisystematic reviews (two assessing dexmedetomidine ${ }^{52} 53$ and one overall alpha-2-agonists ${ }^{48}$ ) did not find evidence of an effect.

Conversely, four semisystematic reviews ${ }^{47-51}$ and a subgroup analysis (including two trials and a total of 415 patients) in a semisystematic review, which assessed alpha-2-agonists in the primary analysis, ${ }^{48}$ found evidence of a beneficial effect of dexmedetomidine compared with different alternative sedatives. ${ }^{47-51}$ In various subgroup analyses (on patients undergoing invasive ventilation, compared with midazolam only, restricted to general ICU), without any adjustment for statistical multiplicity, dexmedetomidine was found to have a beneficial effect for the prevention of delirium..$^{50}$

Quality of life

We did not identify any systematic review or semisystematic review assessing quality of life.

\section{Proportion of participants with non-serious adverse events}

When assessing adverse events, Tran et a $\tilde{l}^{4}$ narratively reported on three trials. Two trials found no evidence of a difference in adverse events comparing dexmedetomidine with propofol, or between dexmedetomidine and midazolam. ${ }^{6162}$ The third trial comparing dexmedetomidine with normal saline found that dexmedetomidine was associated with higher rates of bradycardia, but with lower rates of tachycardia. ${ }^{63}$

\section{Cognitive function}

We did not identify any systematic review or semisystematic review assessing cognitive function.

Additional outcomes reported by the systematic review and the semisystematic reviews

Twenty-three additional outcomes (mainly) on the effect of dexmedetomidine versus other sedatives were reported by the systematic review and semisystematic reviews (supplementary material table 3 ).

\section{Cholinesterase inhibitors}

We did not identify any systematic review or semisystematic review assessing the effects of cholinesterase inhibitors for the prevention of delirium.

\section{Opioids}

We did not identify any systematic review or semisystematic review assessing the effects of opioids for the prevention of delirium.

\section{Melatonine and melatonine inhibitors}

We did not identify any systematic review or semisystematic review assessing the effects of melatonine or melatonine inhibitors for the prevention of delirium.

\section{Management of delirium \\ Antipsychotics}

We did not identify any systematic review or semisystematic review assessing the effects of antipsychotics (eg, haloperidol) for the management of manifest delirium (table 2).

Of all 378 included reviews, $227(60 \%)$ stated that haloperidol was indicated for the management of delirium, 43 $(11 \%)$ stated that haloperidol was contraindicated and 108 (29\%) did not state whether haloperidol was indicated or not. 
Table 3 Summary of findings

\begin{tabular}{|c|c|c|c|c|c|}
\hline $\begin{array}{l}\text { Pharmacological } \\
\text { intervention }\end{array}$ & $\begin{array}{l}\text { No. of systematic } \\
\text { reviews according } \\
\text { to PRISMA with } \\
\text { low risk of bias }\end{array}$ & $\begin{array}{l}\text { No. of systematic } \\
\text { reviews } \\
\text { according to } \\
\text { PRISMA with } \\
\text { high risk of bias }\end{array}$ & $\begin{array}{l}\text { No. of } \\
\text { semisystematic } \\
\text { reviews } \\
\text { according to } \\
\text { PRISMA* }\end{array}$ & $\begin{array}{l}\text { Quality of } \\
\text { the evidence }\end{array}$ & Comments \\
\hline $\begin{array}{l}\text { Delirium } \\
\text { prevention }\end{array}$ & 1 & 0 & 8 & low & $\begin{array}{l}\text { Seven trials with overall } \\
\text { high risk of bias included in } \\
\text { the systematic review with } \\
\text { low risk of bias. The eight } \\
\text { semisystematic reviews were all } \\
\text { high risk of bias and included } \\
\text { solely trials with overall high } \\
\text { risk of bias. }\end{array}$ \\
\hline $\begin{array}{l}\text { Delirium } \\
\text { management }\end{array}$ & 0 & 0 & 0 & No evidence & $\begin{array}{l}\text { No systematic reviews } \\
\text { according to PRISMA were } \\
\text { identified. Neither was a } \\
\text { semisystematic review } \\
\text { identified. }\end{array}$ \\
\hline
\end{tabular}

Presence and quality of evidence by type of pharmacological intervention.

*In agreement with the PRISMA statement except two arbitrary PRISMA criteria.

PRISMA, Preferred Reporting Items for Systematic Reviews and Meta-Analyses.

\section{Sedatives}

We did not identify any systematic review or semisystematic review assessing the effects of sedatives for the management of manifest delirium.

\section{Cholinesterase inhibitors}

We did not identify any systematic review or semisystematic review assessing the effects of cholinesterase inhibitors for the management of manifest delirium.

\section{Opioids}

We did not identify any systematic review or semisystematic review assessing the effects of opioids for the management of manifest delirium.

\section{Melatonine and melatonine inhibitors}

We did not identify any systematic review or semisystematic review assessing the effects of melatonine or melatonine inhibitors for the management of manifest delirium.

\section{DISCUSSION}

\section{Summary of main results}

This overview addresses the evidence for the prevention of delirium and management of manifest delirium with pharmacological agents in ICU patients. We identified only one systematic review ${ }^{46}$ out of a total of 378 reviews which addressed this topic. We classified eight as semisystematic reviews ${ }^{47-53}$ and 369 as narrative reviews. We only found the systematic review to have overall low risk of bias; all eight semisystematic reviews had overall high risk of bias. The identified systematic review with low risk of bias included seven randomised clinical trials ${ }^{55-60}$; which all had overall high risk of bias. Our main results are summarised in the Summary of findings table (table 3).

\section{Strengths and limitations of this study}

This overview of reviews has several methodological strengths. We conducted a comprehensive literature search to identify reviews and meta-analyses in six major electronic databases, with specifically designed search strategies with no limits to publication year, type of publication or language. We used a transparent and systematic method, which was registered and published before the initiation of this project. Each phase of the screening, data extraction, data collection and methodological evaluations were performed by independent review authors working in pairs.

This overview of reviews also has methodological limitations. First, we chose PRISMA as the gold standard for defining a systematic review. One may argue that it is difficult for older reviews to adhere to the PRISMA statement, as this was published in 2009. One may also argue that there may be PRISMA criteria that might not be as important as others, for example, a structured abstract. In contrast, risk of bias evaluation in individual trials is of huge importance for the conclusion of the review. ${ }^{64}$ Therefore, we chose pragmatically to classify all reviews, failing on a maximum of two PRISMA criteria, as semisystematic reviews. Second, we did not search for individual trials to perform a systematic review with meta-analyses and trial sequential analysis within each of the groups of pharmacological agents. Unfortunately, our results revealed that no systematic review on delirium management with any pharmacological agent has been published. Thus, we cannot discuss the evidence on pharmacological prevention or management strategies based on published trials, but merely according to the published reviews. 
Current research within delirium is challenged by methodological and clinical limitations. The main limitations revealed by this overview of reviews is the overall high risk of bias found both in all the semisystematic reviews and all the included trials. It is therefore likely that we purport results that are also biased, that is, beneficial results may be overestimated, and harms may be underestimated. ${ }^{64-66}$ In addition, we found a significant limitation to the research in the ICU delirium field, as systematic reviews adhering (or largely) to the PRISMA criteria all examined dexmedetomidine, which therefore dominates the current literature on pharmacological agents for delirium. Furthermore, the mechanisms of delirium are still not fully established and the underlying cause of delirium in medical ICU patients may be different from those in postoperative ICU patients, suggesting different optimal prevention and management strategies in the mixed ICU population. Certain subgroups of patients with delirium and risk factors at baseline (eg, age, severity of illness, exposure to a surgical procedure, cognitive dysfunction) may influence patient-centred outcomes differently. Current published trials have not stratified according to these factors but may in future research add new knowledge to the ICU field. Another important consideration is that many so-called placebo-controlled trials are not truly placebo-controlled, as some trials include rescue medications like haloperidol 'as needed'.

No study has previously attempted to systematically collect and evaluate all published reviews within pharmacological interventions for delirium. We found that narrative and non-systematic reviews dominate the literature on pharmacological interventions for delirium. Our findings confirm the observations by Siontis $e t a l^{67}$ that publications of erratic quality are produced in massive scales, in publications on the same topic, making it difficult to quickly get an evidence-based insight and overview. Our results reveal that many reviews cite trial results uncritically, leaving readers with the impression that, for example, haloperidol is a proven suitable pharmacological agent for the management of manifest delirium. Rapid access to current research to ensure evidence-based decision making and practice is increasingly demanded by the healthcare system, but guideline developers and decision makers are likely to be overwhelmed by the high numbers of published reviews of erratic quality.

\section{Delirium prevention}

Using a pharmacological delirium prevention protocol in adult ICU patients is not currently recommended. ${ }^{17}$ The identified systematic review and eight semisystematic reviews considered prevention of delirium with dexmedetomidine, when used as a sedative, and found conflicting results, five in favour of dexmedetomidine ${ }^{47-51}$ and three showing equipoise ${ }^{465253}$ results. However, trials with overall high risk of bias and small sample sizes not reaching the required information size in a meta-analysis, ${ }^{68}$ as well as demonstrating huge heterogeneity of unexplained origin, prevent us from presenting any recommendations for the use of dexmedetomidine for the prevention of ICU delirium. We did not find a systematic review or semisystematic review addressing delirium prevention with haloperidol. To our knowledge, 10 randomised trials on haloperidol including a total of 3772 ICU patients or patients having major surgeries have been published. ${ }^{32-34} 69-75$

Sedation trials for the prevention of delirium overshadows research in preventive strategies. However, today, sedation is generally lessened, and light sedation and daily sedative interruption are recommended (low-quality evidence).${ }^{17}$ Sedation with dexmedetomidine and propofol are recommended over benzodiazepines in mechanically ventilated adults (low quality of evidence) ${ }^{17}$; however, no pharmacological agent is recommended for the prevention of delirium. ${ }^{17}$ Patients may presumably benefit from being sedated with an agent which may lower the incidence of delirium, but using an agent to prevent delirium may then compete with the trend of minimising sedation. ${ }^{76}$

\section{Delirium management}

We did not find a systematic review according to the PRISMA criteria addressing pharmacological agents for the management of manifest delirium in ICU patients. To our knowledge, seven randomised trials investigating the effect of haloperidol for the management of manifest delirium in critically ill patients have been published $^{35}$ 77-82 including only a total of 394 critically ill patients. Our overview of reviews demonstrates that the majority of reviews $(60 \%)$, discussing the effect or use of haloperidol for delirium management, cite that haloperidol is indicated, and only $11 \%$ states that haloperidol is contraindicated. For whatever reason, the widespread use and endorsement of haloperidol contradicts the frequent serious adverse reactions shown in other settings, ${ }^{28}$ and the fact that the Food and Drug Administration warns against the use of haloperidol in patients with dementia-related psychosis, because of a 1.6-times increased mortality. ${ }^{83}$

\section{Unanswered questions and future research}

In evidence-based medicine, systematic reviews of randomised trials rank highest. However, systematic reviews must be performed based on methods aiming to minimise systematic and random errors; otherwise, the results will be questionable. In addition to a thorough and systematic bias risk assessment, meta-analysis needs to reach a required information size (meta-analytic sample size) based on a minimal important clinical difference to conclude whether an intervention is better than another. Otherwise, a conclusion based on meta-analyses with high risk of random error ${ }^{45} 6584$ may be communicated. The lack of evidence and poor quality of the present evidence on the use of pharmacological agents for delirium leave clinicians to decide which pharmacological intervention to use. Research on how to deal with the management of manifest delirium, when all non-pharmacological 
options have been used, is highly warranted. Although multicomponent, non-pharmacological intervention focusing on reducing modifiable risk factors for delirium, improving cognition and optimising sleep, mobility, hearing, and vision in critically ill adults, as well as early mobilisation, is recommended to reduce the incidence and duration in the ICU, this is only supported by low quality of evidence. ${ }^{17}$ In settings outside the ICU, non-pharmacological multicomponent protocols have shown promising results (moderate level of quality). ${ }^{85} 86$ However, such multifaceted interventions have not been adequately studied in the ICU setting. Based on the available evidence, one might get the idea that there is some evidence for the effect of dexmedetomidine to prevent delirium. However, as our overview underlines, there is really no valid evidence to support the use of dexmedetomidine and none at all that dexmedetomidine is better than haloperidol (or vice versa), which seems to be the preferred agent so far. ${ }^{16} 19$

\section{CONCLUSION}

Our overview of reviews demonstrated that systematic reviews and semisystematic reviews currently available in the delirium literature are heterogeneous in quality with high risk of bias. The results were conflicting regarding the effect of dexmedetomidine for the prevention of delirium based on the high-quality systematic review and the semisystemtic reviews. There is no evidence for the use of any pharmacological agent for the management of manifest delirium based on systematic or semisystematic reviews.

There is an urgent need for a systematic review with low risk of bias assessing the effects of pharmacological prevention of delirium and management of manifest delirium in ICU patients. Especially the effects of haloperidol need to be assessed, because haloperidol is the most recommended drug for the management of delirium. Future systematic reviews should aim to adhere to the PRISMA statement, so risk of systematic errors is minimised, and the best available evidence is presented. Furthermore, future trials on any antidelirious agent should report on patient-centred outcomes.

Identifying the most effective intervention for both the prevention of delirium and management of manifest delirium in ICU patients will benefit patients, relatives and healthcare systems around the world.

\section{Difference between protocol and review}

In our published protocol which was written a priori initiation of the overview, we stated that we would categorise reviews into the following groups: (1) systematic reviews according to PRISMA with low risk of bias assessed with ROBIS; (2) systematic reviews according to PRISMA with high risk of bias assessed with ROBIS; and (3) non-systematic reviews according to PRISMA.

Because we only found one systematic review fulfilling all the PRISMA criteria, we decided post protocol publication to acknowledge reviews almost fulfilling the PRISMA criteria by adding the category semisystematic reviews.

\section{Author affiliations}

${ }^{1}$ Copenhagen Trial Unit, Centre for Clinical Intervention Research, Rigshospitalet, Copenhagen University Hospital, Copenhagen, Denmark

${ }^{2}$ Centre for Research in Intensive Care, Rigshospitalet, Copenhagen University Hospital, Copenhagen, Denmark

${ }^{3}$ Department of Intensive Care, Rigshospitalet, Copenhagen University Hospital, Copenhagen, Denmark

${ }^{4}$ Department of of Neuroanaesthesiology, Rigshospitalet, Copenhagen University hospital, Copenhagen, Denmark

${ }^{5}$ Department of Cardiology, Holbaek Hospital, Holbaek, Denmark

Acknowledgements We thank Dezhao Kong, Ning Lianhy, Di Wang and Sainan Fang for helping with the translation of the Chinese reviews.

Contributors Coordinating the review: MB. Conception and design of the review: MB, JCJ, AP, JW. Performing search strategies and literature searches: MB. Performing the literature screening: MB, SRK, LKL, MOC. Performing the data extraction: MB, SRK, LKL, MOC. Writing the review: MB, JW, JCJ, AP, MOC, LKL, SRK. All authors critically reviewed and revised the manuscript and approved the final version for publication. MB is the guarantor of the review.

Funding MB, MOC, JCJ, AP and JW were supported by the public Innovation Fund Denmark (4108-00011B), which did not have any role in study design, data collection, data analysis, data interpretation or writing of the report. No other sources of financial support were obtained for this review.

Competing interests MB, MOC, JCJ, AP and JW had financial support from the public Innovation Fund Denmark; AP is head of research at the Dept. of Intensive Care unit at Rigshospitalet. The department receives support for research from CSL Behring, Fresenius Kabi and Ferring Pharmaceuticals. AP and JW are members of the AID-ICU steering group (NCT03392376); no other relationships or activities that could appear to have influenced the submitted work.

Patient consent for publication Not required.

Provenance and peer review Not commissioned; externally peer reviewed. Data sharing statement № additional data are available.

Open access This is an open access article distributed in accordance with the Creative Commons Attribution Non Commercial (CC BY-NC 4.0) license, which permits others to distribute, remix, adapt, build upon this work non-commercially, and license their derivative works on different terms, provided the original work is properly cited, appropriate credit is given, any changes made indicated, and the use is non-commercial. See: http://creativecommons.org/licenses/by-nc/4.0/.

\section{REFERENCES}

1. American Psychiatric Association. Practice Guidelines for the treatment of psychiatric disorders, 2006.

2. Peterson JF, Pun BT, Dittus RS, et al. Delirium and its motoric subtypes: a study of 614 critically ill patients. J Am Geriatr Soc 2006;54:479-84.

3. Pandharipande P, Cotton BA, Shintani A, et al. Motoric subtypes of delirium in mechanically ventilated surgical and trauma intensive care unit patients. Intensive Care Med 2007;33:1726-31.

4. Meagher DJ, Trzepacz PT. Motoric subtypes of delirium. Semin Clin Neuropsychiatry 2000;5:75-85.

5. Ely EW, Inouye SK, Bernard GR, et al. Delirium in mechanically ventilated patients: validity and reliability of the confusion assessment method for the intensive care unit (CAM-ICU). JAMA 2001;286:2703-10.

6. Ely EW, Gautam S, Margolin R, et al. The impact of delirium in the intensive care unit on hospital length of stay. Intensive Care Med 2001;27:1892-900.

7. Ely EW, Girard TD, Shintani AK, et al. Apolipoprotein E4 polymorphism as a genetic predisposition to delirium in critically ill patients. Crit Care Med 2007;35:112-7.

8. Hipp DM, Ely EW. Pharmacological and nonpharmacological management of delirium in critically ill patients. Neurotherapeutics 2012;9:158-75. 
9. Inouye SK, Westendorp RG, Saczynski JS. Delirium in elderly people. Lancet 2014;383:911-22.

10. Milbrandt EB, Deppen S, Harrison PL, et al. Costs associated with delirium in mechanically ventilated patients. Crit Care Med 2004;32:955-62.

11. Girard TD, Jackson JC, Pandharipande PP, et al. Delirium as a predictor of long-term cognitive impairment in survivors of critical illness. Crit Care Med 2010;38:1513-20.

12. Jackson JC, Gordon SM, Hart RP, et al. The association between delirium and cognitive decline: a review of the empirical literature. Neuropsychol Rev 2004;14:87-98.

13. Lin SM, Liu CY, Wang CH, et al. The impact of delirium on the survival of mechanically ventilated patients. Crit Care Med 2004;32:2254-9.

14. Pisani MA, Kong SY, Kasl SV, et al. Days of delirium are associated with 1-year mortality in an older intensive care unit population. $\mathrm{Am} \mathrm{J}$ Respir Crit Care Med 2009;180:1092-7.

15. van den Boogaard M, Schoonhoven L, Evers AW, et al. Delirium in critically ill patients: impact on long-term health-related quality of life and cognitive functioning. Crit Care Med 2012;40:112-8.

16. Collet MO, Caballero J, Sonneville R, et al. Prevalence and risk factors related to haloperidol use for delirium in adult intensive care patients: the multinational AID-ICU inception cohort study. Intensive Care Med 2018;44:1081-9.

17. Devlin JW, Skrobik Y, Gélinas C, et al. Clinical practice guidelines for the prevention and management of pain, agitation/sedation, delirium, immobility, and sleep disruption in adult patients in the ICU. Crit Care Med 2018;46:e825-e873.

18. Bannon L, McGaughey J, Clarke M, et al. Impact of nonpharmacological interventions on prevention and treatment of delirium in critically ill patients: protocol for a systematic review of quantitative and qualitative research. Syst Rev 2016;5:75.

19. Borthwick MBR, Craig M, Egan A, et al. Detection, prevention and treatment of delirium in critically ill patients. Intensive Care Society $2006 \mathrm{http}: / /$ members.ics.ac.uk/AsiCommon/Controls/BSA/ Downloader.aspx?iDocumentStorageKey=d616cece-070a-48a4bfaa-18da5a546634\&iFileTypeCode=PDF\&iFileName=Detection, $\%$ 20Prevention\%20and\%20Treatment\%20of\%20Delirium (Accessed Mar 2018)

20. British Geriatrics Society. The prevention, diagnosis and management of delirium in older people - National guideline. 2006 https://www.rcplondon.ac.uk/guidelines-policy/prevention-diagnosisreferral-and-management-delirium-older-people (Accessed Mar 2018)

21. Mac Sweeney R, Barber V, Page V, et al. A national survey of the management of delirium in UK intensive care units. QJM 2010;103:243-51.

22. Devlin JW, Bhat S, Roberts RJ, et al. Current perceptions and practices surrounding the recognition and treatment of delirium in the intensive care unit: a survey of 250 critical care pharmacists from eight states. Ann Pharmacother 2011;45:1217-29.

23. George SPC, Patteril MV. Managing delirium in Intensive care. Intensive Care Medicine 2009;35(1 supplement):s258.

24. Meyer AMA, Dornblaser EK, Bellamy CJ, et al. A multi-center characterization of antipsychotic use for the treatment of delirium in medical ICU patients. Pharmacotherapy 2011;31:421e.

25. Trogrlic Z, Ista E, Slooter A, Bakker J, et al. Correction: Current practices in ICU delirium management: a prospective multicenter study in the Netherlands. Critical Care 2013;17:P548.

26. Martin J, Heymann A, Bäsell K, et al. Evidence and consensus-based German guidelines for the management of analgesia, sedation and delirium in intensive care-short version. Ger Med Sci 2010;8:Doc02.

27. Dansk Selskab for Anæstesi og Intensiv Medicin (DASAIM). Sedationsstrategi - Målrettet behandling af gener forbundet med kritisk sygdom. $2014 \mathrm{https} / /$ www.google.dk/url?sa=t\&rct=j\&q=\& esrc $=$ s\&source $=$ web\&cd $=1$ \&ved $=0$ ahUKEwjkz_yS4cXOAhWF1i wKHQySCpMQFggcMAA\&url=http\%3A\%2F\%2Fwww.dasaim.dk\% 2Fwp-content\%2Fuploads\%2F2015\%2F09\%2FSedationsstrategisept15.pdf\&usg=AFQjCNF9xGfr3aoeEBMTTPHrDpBm2BqMSw\& cad=rja (Accessed Mar 2018).

28. Barr J, Fraser GL, Puntillo K, et al. Clinical practice guidelines for the management of pain, agitation, and delirium in adult patients in the intensive care unit. Crit Care Med 2013;41:263-306.

29. Grimshaw JM, Santesso N, Cumpston M, et al. Knowledge for knowledge translation: the role of the Cochrane Collaboration. $J$ Contin Educ Health Prof 2006;26:55-62.

30. Laupacis A, Straus S. Systematic reviews: time to address clinical and policy relevance as well as methodological rigor. Ann Intern Med 2007;147:273-4.

31. Devlin JW, Roberts RJ, Fong JJ, et al. Efficacy and safety of quetiapine in critically ill patients with delirium: a prospective, multicenter, randomized, double-blind, placebo-controlled pilot study. Crit Care Med 2010;38:419-27.

32. Reade MC, O'Sullivan K, Bates S, et al. Dexmedetomidine vs. haloperidol in delirious, agitated, intubated patients: a randomised open-label trial. Crit Care 2009;13:R75

33. Girard TD, Pandharipande PP, Carson SS, et al. Feasibility, efficacy, and safety of antipsychotics for intensive care unit delirium: the MIND randomized, placebo-controlled trial. Crit Care Med 2010;38:428-37.

34. Page VJ, Ely EW, Gates S, et al. Effect of intravenous haloperidol on the duration of delirium and coma in critically ill patients (Hope-ICU): a randomised, double-blind, placebo-controlled trial. Lancet Respir Med 2013;1:515-23.

35. van Eijk MM, Roes KC, Honing ML, et al. Effect of rivastigmine as an adjunct to usual care with haloperidol on duration of delirium and mortality in critically ill patients: a multicentre, double-blind, placebocontrolled randomised trial. Lancet 2010;376:1829-37.

36. Barbateskovic M, Larsen LK, Oxenbøll-Collet M, et al. Pharmacological interventions for delirium in intensive care patients: a protocol for an overview of reviews. Syst Rev 2016;5:211.

37. Higgins J, Green S, eds. Cochrane Handbook for Systematic Reviews of Interventions Version 5.1.0 [updated March 2011]: The Cochrane Collaboration, 2011.

38. Robinson KA, Chou R, Berkman ND, et al. Twelve recommendations for integrating existing systematic reviews into new reviews: EPC guidance. J Clin Epidemiol 2016;70:38-44.

39. Liberati A, Altman DG, Tetzlaff J, et al. The PRISMA statement for reporting systematic reviews and meta-analyses of studies that evaluate healthcare interventions: explanation and elaboration. $B M J$ 2009;339:b2700.

40. International Council for Harmonisation of Technical Requirements for pharmaceuticals for human use (ICH). Guideline for Good Clinical Practice E6(R2), 2016.

41. Ware JE, Sherbourne CD. The MOS 36-item short-form health survey (SF-36). I. Conceptual framework and item selection. Med Care 1992;30:473-83.

42. Randolph C, Tierney MC, Mohr E, et al. The Repeatable Battery for the Assessment of Neuropsychological Status (RBANS): preliminary clinical validity. J Clin Exp Neuropsychol 1998;20:310-9.

43. Veritas Health Innovation. Covidence systematic review software. Melbourne, Australia, 2018. https://www.covidence.org/. (Accessed Mar 2018).

44. Whiting P, Savović J, Higgins JP, et al. ROBIS: A new tool to assess risk of bias in systematic reviews was developed. J Clin Epidemiol 2016;69:225-34.

45. Wetterslev J, Thorlund K, Brok J, et al. Trial sequential analysis may establish when firm evidence is reached in cumulative meta-analysis. $J$ Clin Epidemiol 2008;61:64-75.

46. Chen K, Lu Z, Xin YC, et al. Alpha-2 agonists for long-term sedation during mechanical ventilation in critically ill patients. Cochrane Database Syst Rev 2015;1:Cd010269.

47. Xia ZQ, Chen $S Q$, Yao X, et al. Clinical benefits of dexmedetomidine versus propofol in adult intensive care unit patients: a meta-analysis of randomized clinical trials. J Surg Res 2013;185:833-43

48. Zhang H, Lu Y, Liu M, et al. Strategies for prevention of postoperative delirium: a systematic review and meta-analysis of randomized trials. Crit Care 2013;17:R47.

49. Liu X, Xie G, Zhang K, et al. Dexmedetomidine vs propofol sedation reduces delirium in patients after cardiac surgery: A meta-analysis with trial sequential analysis of randomized controlled trials. J Crit Care 2017:38:190-6.

50. Pasin L, Landoni G, Nardelli P, et al. Dexmedetomidine reduces the risk of delirium, agitation and confusion in critically III patients: a meta-analysis of randomized controlled trials. J Cardiothorac Vasc Anesth 2014;28:1459-66.

51. Lin YY, He B, Chen J, et al. Can dexmedetomidine be a safe and efficacious sedative agent in post-cardiac surgery patients? a metaanalysis. Crit Care 2012;16:R169.

52. Fraser GL, Devlin JW, Worby CP, et al. Benzodiazepine versus nonbenzodiazepine-based sedation for mechanically ventilated, critically ill adults: a systematic review and meta-analysis of randomized trials. Crit Care Med 2013:41(9 Suppl 1):S30-8.

53. Tan JA, Ho KM, Km H. Use of dexmedetomidine as a sedative and analgesic agent in critically ill adult patients: a meta-analysis. Intensive Care Med 2010;36:926-39.

54. Tran A, Blinder H, Hutton B, et al. A systematic review of alpha-2 agonists for sedation in mechanically ventilated neurocritical care patients. Neurocrit Care 2018;28:12-25.

55. Jakob SM, Ruokonen E, Grounds RM, et al. Dexmedetomidine vs midazolam or propofol for sedation during prolonged 
mechanical ventilation: two randomized controlled trials. JAMA 2012;307:1151-60.

56. Pandharipande PP, Pun BT, Herr DL, et al. Effect of sedation with dexmedetomidine vs lorazepam on acute brain dysfunction in mechanically ventilated patients: the MENDS randomized controlled trial. JAMA 2007;298:2644-53.

57. Riker RR, Shehabi Y, Bokesch PM, et al. Dexmedetomidine vs midazolam for sedation of critically ill patients: a randomized trial. JAMA 2009:301:489-99.

58. Ruokonen E, Parviainen I, Jakob SM, et al. Dexmedetomidine versus propofol/midazolam for long-term sedation during mechanical ventilation. Intensive Care Med 2009;35:282-90.

59. Shehabi Y, Bellomo R, Reade MC, et al. Early goal-directed sedation versus standard sedation in mechanically ventilated critically ill patients: a pilot study*. Crit Care Med 2013;41:1983-91.

60. Xu JB WY, Shi QS. A combined protocol for dexmedetomidine used in prolonged sedation in intensive care unit. Modern Medicine Journal of China 2012;14:20-2.

61. Srivastava VK, Agrawal S, Kumar S, et al. Comparison of dexmedetomidine, propofol and midazolam for short-term sedation in postoperatively mechanically ventilated neurosurgical patients. $J$ Clin Diagn Res 2014;8:Gc04-7.

62. Mirski MA, Lewin JJ, Ledroux S, et al. Cognitive improvement during continuous sedation in critically ill, awake and responsive patients: the Acute Neurological ICU Sedation Trial (ANIST). Intensive Care Med 2010;36:1505-13.

63. Zhao LH, Shi ZH, Chen GQ, et al. USe of dexmedetomidine for prophylactic analgesia and sedation in patients with delayed extubation after craniotomy: A randomized controlled trial. $J$ Neurosurg Anesthesiol 2017;29:132-9.

64. Savović J, Jones HE, Altman DG, et al. Influence of reported study design characteristics on intervention effect estimates from randomized, controlled trials. Ann Intern Med 2012;157:429-38.

65. Jakobsen JC, Wetterslev J, Winkel P, et al. Thresholds for statistical and clinical significance in systematic reviews with meta-analytic methods. BMC Med Res Methodol 2014;14:120.

66. Lundh A, Lexchin J, Mintzes B, et al. Industry sponsorship and research outcome. Cochrane Database Syst Rev 2017;2:Mr000033.

67. Siontis KC, Hernandez-Boussard T, loannidis JP. Overlapping meta-analyses on the same topic: survey of published studies. BMJ 2013;347:f4501.

68. Wetterslev J, Thorlund K, Brok J, et al. Estimating required information size by quantifying diversity in random-effects model meta-analyses. BMC Med Res Methodol 2009;9:86.

69. Wang W, Li HL, Wang DX, et al. Haloperidol prophylaxis decreases delirium incidence in elderly patients after noncardiac surgery: a randomized controlled trial* Crit Care Med 2012:40:731-9.

70. Kalisvaart KJ, de Jonghe JF, Bogaards MJ, et al. Haloperidol prophylaxis for elderly hip-surgery patients at risk for delirium: a randomized placebo-controlled study. J Am Geriatr Soc 2005;53:1658-66.

71. van den Boogaard $\mathrm{M}$, Schoonhoven $\mathrm{L}$, van Achterberg $\mathrm{T}$, et al. Haloperidol prophylaxis in critically ill patients with a high risk for delirium. Crit Care 2013;17:R9.
72. Kaneko TCJ, Ishikura T, Kobayashi M, et al. Prophylactic consecutive administration of haloperidol can reduce the occurrence of postoperative delirium in gastrointestinal surgery. Yonago Acta medica 1999;42:179-84.

73. Fukata S, Kawabata Y, Fujisiro K, et al. Haloperidol prophylaxis does not prevent postoperative delirium in elderly patients: a randomized, open-label prospective trial. Surg Today 2014:44:2305-13.

74. Al-Qadheeb NS, Skrobik Y, Schumaker G, et al. Preventing ICU subsyndromal delirium conversion to delirium with low-dose iv haloperidol: A double-blind, placebo-controlled pilot study. Crit Care Med 2016;44:583-91.

75. van den Boogaard M, Slooter AJC, Brüggemann RJM, et al. Effect of Haloperidol on Survival Among Critically III Adults With a High Risk of Delirium. JAMA 2018;319:680-90.

76. Strøm T, Martinussen T, Toft P. A protocol of no sedation for critically ill patients receiving mechanical ventilation: a randomised trial. Lancet 2010;375:475-80.

77. Skrobik YK, Bergeron N, Dumont M, et al. Olanzapine vs haloperidol: treating delirium in a critical care setting. Intensive Care Med 2004;30:444-9.

78. Atalan N, Efe Sevim M, Akgün $\mathrm{S}$, et al. Morphine is a reasonable alternative to haloperidol in the treatment of postoperative hyperactive-type delirium after cardiac surgery. $J$ Cardiothorac Vasc Anesth 2013:27:933-8.

79. Tagarakis GI, Voucharas C, Tsolaki F, et al. Ondasetron versus haloperidol for the treatment of postcardiotomy delirium: a prospective, randomized, double-blinded study. J Cardiothorac Surg 2012;7:25.

80. Breitbart W, Marotta R, Platt MM, et al. A double-blind trial of haloperidol, chlorpromazine, and lorazepam in the treatment of delirium in hospitalized AIDS patients. Am J Psychiatry 1996;153:231-7.

81. Lin CJ SF, Fang CK, Chen HW, et al. An open trial comparing haloperidol with olanzapine for the treatment of delirium in palliative and hospice center cancer patients. Journal of Internal Medicine of Taiwan 2008;19:346-54.

82. Han CS, Kim YK. A double-blind trial of risperidone and haloperido for the treatment of delirium. Psychosomatics 2004;45:297-301.

83. Food and Drug Administration (FDA), 2005. https://www.google.dk/ url? sa $=$ t\&rct $=j \& q=\& e s r c=s \&$ source $=$ web\& $c d=1 \& v e d=0$ ahUKEwj $J$ 6eB07nQAhVF1xoKHXJyCP8QFggcMAA\&url=http\%3A\%2F\% 2Fwww.accessdata.fda.gov\%2Fdrugsatfda docs\%2Flabel\% 2F2008\%2F015923s082\%2C018701s057lbl.pdf\&usg=AFQjCNGb dzQFtVi2X5DcDmVDGRFmnujVYQ\&cad=rja (Accessed Mar 2018).

84. Turner RM, Bird SM, Higgins JP. The impact of study size on metaanalyses: examination of underpowered studies in Cochrane reviews. PLoS One 2013;8:e59202.

85. Abraha I, Trotta F, Rimland JM, et al. Efficacy of NonPharmacological Interventions to Prevent and Treat Delirium in Older Patients: A Systematic Overview. The SENATOR project ONTOP Series. PLoS One 2015;10:e0123090.

86. Abraha I, Rimland JM, Trotta F, et al. Non-pharmacological interventions to prevent or treat delirium in older patients: Clinical practice recommendations the SENATOR-ONTOP series. J Nutr Health Aging 2016;20:927-36. 